\title{
Escenarios financieros para la dinamización turística del Parque Nacional Cotopaxi
}

\section{Financial scenarios for the tourist revitalization of the Cotopaxi National Park}

Fabián Camilo Tapia Novillo ${ }^{1 *}$, Jimena de los Ángeles Chango Yanza ${ }^{2}$, Patricia Mercedes Pazmiño Valle ${ }^{3}$

${ }^{1}$ Universidad Central del Ecuador, Facultad de Ciencias Agrícolas, Jerónimo Leyton y Gato Sobral, 170521, Quito, Ecuador. 凶اfctapia@uce.edu.ec.

https://orcid.org/0000-0003-3669-7009

${ }^{2}$ Universidad Central del Ecuador, Facultad de Ciencias Agrícolas, Jerónimo Leyton y Gato Sobral, 170521, Quito, Ecuador. 凶ijchango@uce.edu.ec.

${ }^{3}$ Universidad Central del Ecuador, Facultad de Ciencias Agrícolas, Jerónimo Leyton y Gato Sobral, 170521, Quito, Ecuador. 凶pmpazmino@uce.edu.ec.

\section{Resumen}

La insuficiencia de recursos económicos, conjugado con un estatismo centralizado, convergen en un escaso desarrollo de los destinos turísticos. El objetivo del estudio fue determinar los escenarios financieros óptimos para la dinamización turística del Parque Nacional Cotopaxi. Mediante un estudio transversal descriptivo, se aplicó un muestreo aleatorio simple sistémico a una muestra representativa de visitantes al destino. Se determinó tres escenarios financieros, el precio de la tarifa al usuario nacional en cinco dólares, e internacional en diez dólares, y los elementos fundamentales para la dinamización turística. La autogestión financiera marcará un hito turístico para la independencia de un régimen estatal centralista hacia la dinamización turística de un destino.

Palabras clave: áreas nacionales protegidas, escenarios financieros, auto sostenibilidad financiera, dinamización turística, Parque Nacional Cotopaxi.

\begin{abstract}
Insufficient economic resources, combined with a centralized statism, converge in a scarce development of tourist destinations. The objective of the study was to determine the optimal financial scenarios for the tourist revitalization of Cotopaxi National Park. Through a descriptive cross-sectional study, a simple systemic random sampling was applied to a representative sample of visitors to the destination. Three financial scenarios were determined, the price of the national user rate in five dollars, and international in ten dollars, and the fundamental elements for the revitalization of tourism. Financial self-management will mark a tourist milestone for the independence of a centralist state regime towards the tourist revitalization of a destination.
\end{abstract}

Keywords: national protected areas, financial scenarios, financial self-sustainability, tourist revitalization, Cotopaxi National Park. 


\section{Introducción}

En el marco de la Constitución de la República del Ecuador, Art. 71 (2008), "la naturaleza o Pacha Mama, donde se reproduce y realiza la vida, tiene derecho a que se respete integralmente su existencia, y el mantenimiento y regeneración de sus ciclos vitales, estructura, funciones y procesos evolutivos". También, se establece que "El Estado asignará los recursos económicos necesarios para la sostenibilidad financiera del sistema [nacional de áreas protegidas], y fomentará la participación de las comunidades, pueblos y nacionalidades que han habitado ancestralmente las áreas protegidas en su administración y gestión" (Art. 405). En esta línea, la Ley de Turismo (2014), establece que "Será competencia de los Ministerios de Turismo y del Ambiente, coordinar el ejercicio de las actividades turísticas en las áreas naturales protegidas, [emitir] las regulaciones o limitaciones de uso por parte de los turistas; la fijación y cobro de tarifas por el ingreso, y demás aspectos relacionados con las áreas naturales protegidas" (Art. 20). El Plan Nacional de Desarrollo 2017-2021-Toda una Vida plantea como objetivo "Posicionar y potenciar al Ecuador como un país megadiverso, intercultural y multiétnico, desarrollando y fortaleciendo la oferta turística nacional y las industrias culturales; fomentando el turismo receptivo como fuente generadora de divisas y empleo, en un marco de protección del patrimonio natural y cultural" (Senplades, 2017, p. 106). En el mismo documento se plantea como meta para el año 2021, "Incrementar el ingreso de divisas por concepto de turismo receptor de US\$ $1,4 \mathrm{~mm}$ a US\$ 2,5 mm" (p. 106)

Las áreas protegidas (AP) surgen en el siglo pasado en Estados Unidos de Norteamérica con la creación del Parque Nacional Yellowstone, como mecanismo para salvaguardar elementos naturales y culturales permanentes (Cifuentes et al., 2000). Se define a las áreas protegidas como: "un espacio geográfico claramente definido, reconocido, dedicado y gestionado, mediante medios legales u otros tipos de medios eficaces para conseguir la conservación a largo plazo de la naturaleza y de sus servicios ecosistémicos y sus valores culturales asociados" (Dudley, 2008, p. 69). Un Parque Nacional es una extensión de tierra que protege la integridad ecológica de uno o más ecosistemas para las generaciones presentes y futuras; excluye la explotación u ocupación no li- gadas a la protección del área, provee las bases para que los visitantes puedan hacer uso espiritual, científico, educacional o recreativo de forma compatible con la preservación y la cultura (Dudley, 2008; Lausche, 2011).

En Ecuador, para una administración eficiente de las áreas protegidas se creó el Sistema Nacional de Áreas Protegidas (SNAP) como el conjunto de áreas naturales que garantizan la cobertura y conectividad de ecosistemas importantes en los niveles terrestre, marino y costero marino, de sus recursos culturales y de las principales fuentes hídricas (Constitución de la República del Ecuador, Art. 37, 2008; Ministerio del Ambiente [MAE], 2015b). Existen 56 áreas protegidas, lo cual representa el $20 \%$ del territorio nacional, éstas se encuentran distribuidas en las 4 regiones del país; muchas de ellas ocupan más de una provincia, ya que un área protegida es un espacio geográfico claramente definido, reconocido y gestionado mediante medios legales $u$ otros tipos de medios eficaces para conseguir la conservación a largo plazo del área y los servicios ecosistémicos y culturales asociados (MAE, 2018).

Para enrolar las áreas protegidas al turismo, el Patrimonio de Áreas Naturales del Estado (PANE) establece que las actividades de uso público y turístico fomentarán un turismo sostenible. Además, que la planificación, ejecución y control de las modalidades de operación turística serán: investigación, recuperación de áreas ecológicas afectadas, educación ambiental, turismo de naturaleza, turismo de aventura, turismo cultural, ecoturismo (Reglamento especial de turismo en áreas naturales protegidas, 2016). Por tal razón, el Ministerio del Ambiente como una manera de incentivar el turismo, decretó que el ingreso a las áreas protegidas sea gratuito, excepto las islas Galápagos. Esta iniciativa está vigente desde el 17 de enero de 2012, luego de firmar el Acuerdo Ministerial 006. A pesar del decreto de ingreso gratuito a las áreas protegidas, esto depende de las condiciones especiales de cada lugar, para lo cual se respetarán los horarios establecidos y normas vigentes en cada una de estas áreas, además que es gratuito solo el ingreso a las áreas, pero aquellos costos que tienen que ver con desarrollo de investigaciones y filmaciones con fines científicos o periodísticos siguen vigentes y están establecidos en el texto unificado de legislación ambiental, así también los 
costos por servicios turísticos como: cabañas, zonas de camping, servicios de guías naturalistas, los cuales no han variado (MAE, 2018).

Una de las principales áreas protegidas del Ecuador es el Parque Nacional Cotopaxi, el cual está ubicado en las provincias de Cotopaxi, Pichincha y Napo, posee una extensión de 33.393 hectáreas, su año de creación fue 1975, su principal atractivo es el volcán Cotopaxi, uno de los volcanes activos más altos del mundo con 5.897 m s.n.m., el cual domina todo el paisaje del área protegida, donde se encuentran otras dos elevaciones, el Morurco con $4.880 \mathrm{~m}$ s.n.m. y el Rumiñahui con 4.722 m s.n.m (MAE, 2015b). Según el MAE (2019), el número de visitantes entre nacionales y extranjeros que arribaron al Parque Nacional Cotopaxi [PNC] fue de 153.611 en el año 2011, 168.499 en el año 2012 y 233.181 en el año 2018, datos que permiten ver un incremento notable e importante de turistas, cuyo porcentaje entre el año 2011 y 2018 oscila en un $51.8 \%$.

Con este crecimiento vertiginoso de visitantes también aumentaron las necesidades y la brecha de financiamiento del SNAP, el cual determina que el gasto total anual del 2012 bordeó los USD 21,6 millones con un aporte estatal del $94 \%$, y establece una meta óptima financiera de USD 44,1 millones para alcanzar un escenario de consolidación y USD 66,8 millones para lograr un escenario de manejo ideal. Pero para el año 2015 el SNAP contaba con el $48 \%$ del total de los recursos necesarios para alcanzar su consolidación, mientras que para el escenario ideal contaba apenas con el $32 \%$ (MAE, 2015a).

Estudios anteriores determinan el inicio de las áreas protegidas en las culturas de la India y Grecia, que establecieron reservas naturales para proteger paisajes únicos; sin embargo, fue la creación del Parque Nacional de Yellowstone en Estados Unidos en 1872, el evento que marca el desarrollo de reservas para la conservación en los tiempos modernos (Pauchard \& Villarroel, 2002) y constituye el inicio de sistemas de protección frente a la acción humana. A través del tiempo, el Parque Nacional Yellowstone sigue siendo uno de los referentes como destino turístico con una excelente reputación y una percepción positiva del turista, debido a que el visitante tiene todas las facilidades turísticas, infraestructura, planta turística entre otras cosas, que lo hacen atrac- tivo al mercado y por las cuales se cobra una entrada que sirve como fuente de financiamiento (Rivera \& Vallejos-Romero, 2015). Sin una tarifa de ingreso y un olvido de los gobiernos, se puede llegar a poner en riesgo la perpetuidad de las áreas protegidas, debido a la falta de recursos financieros, humanos y logísticos, como lo destacan las investigaciones en el Parque Nacional La Tigra, Tegucigalpa, Honduras (Maldonado, 2000).

La importancia de plantear escenarios financieros como una secuencia hipotética de eventos construidos con el propósito de centrar la atención en los procesos causales y la toma de decisiones con la visualización del futuro de la ANP, parques nacionales, reservas ecológicas, etc., está dado por la exploración de eventos posibles donde los escenarios hacen parte de variados métodos para prever el futuro en una lógica racional con el objetivo de reducir la incertidumbre (Vergara et al. 2010).

La dinamización turística fue tratada por primera vez en la Conferencia Sectorial del Turismo de 1996, teniendo como finalidad los destinos turísticos en fase de desarrollo incipiente, cuyo objetivo es acelerar el crecimiento y asegurar la sostenibilidad futura, donde los beneficiarios son pequeñas poblaciones, ciudades medianas y áreas territoriales extensas (Román, 2011). Las áreas protegidas no pueden quedar al margen, es así que desde la década de los 90 se ha observado en Chile un importante aumento de las áreas protegidas privadas, fenómeno que ocurre en forma espontánea y a pesar de la ausencia de incentivos o de una política que promueva su desarrollo, éstas han implementado con éxito mecanismos de autofinanciamiento basados en el concepto de retribución por servicios ecosistémicos, que pueden ser ampliados socialmente y fortalecidos legalmente (Sepúlveda \& Villarroel, 2006). En esa misma línea, Costa Rica ha optado por el ecoturismo en todas las políticas de desarrollo turístico de forma sustentable, para lo cual ha sido necesario que las áreas protegidas sobre las que se apoya la actividad turística tengan una gestión activa, es decir, cuenten con un plan de manejo vigente y dispongan de recursos económicos y humanos suficientes (Nel-lo Andreu, 2008).

El problema de estudio se fundamenta en las referencias citadas y, contrastándolo con la realidad actual, se puede evidenciar que se tiene dependencia financiera de fondos públicos. La falta de oferta tu- 
rística, infraestructura básica, señalética pobre, falta de sostenibilidad financiera a largo plazo, personal insuficiente o poco capacitado, entre otros, es lo que induce a un desplazamiento del turista, y si se desplaza no provoca el retorno.

Estas cifras, metas y antecedentes hacen imprescindible un estudio de los escenarios financieros de las AP, en este caso del PNC, ya que a pesar de las políticas y leyes, éstas no son consecuentes con la realidad, puesto que es competencia de las autoridades de turismo establecer tarifas de ingreso para las diferentes AP, el manejo de éstas está a cargo del MAE; además, después del decreto de gratuidad del 2012 a las AP, se incurre en la dependencia casi total del financiamiento público, pero esto es insuficiente para el correcto desarrollo del PNC respecto al turismo, puesto que solo se estaría hablando de preservación de AP y no así de conservación de la misma con un modelo de turismo adecuado y específico, donde existan las facilidades y oferta turística plena que cumpla con las expectativas del visitante.
Con los antecedentes expuestos, el objetivo fue determinar los escenarios financieros óptimos para una autosustentabilidad financiera y, en consecuencia, concluir si el visitante está de acuerdo en pagar una tarifa de ingreso, de cuánto sería ésta y qué actividades turísticas le gustaría que se brinden en el parque nacional.

\section{Materiales y métodos}

El Parque Nacional Cotopaxi está ubicado en las provincias de Cotopaxi, Pichincha y Napo (Figura 1), posee una extensión de 33.393 hectáreas, y su año de creación fue 1975 (MAE, 2015b). Su principal atractivo es el volcán Cotopaxi, uno de los volcanes activos más altos del mundo con $5.897 \mathrm{~m}$ s.n.m., el cual maravilla por su forma perfectamente cónica, su manto de nieves perpetuas y una enorme pared de roca negra que parece un ojo, visible desde el norte. El cráter mide 800 metros de diámetro y 334 metros de profundidad.

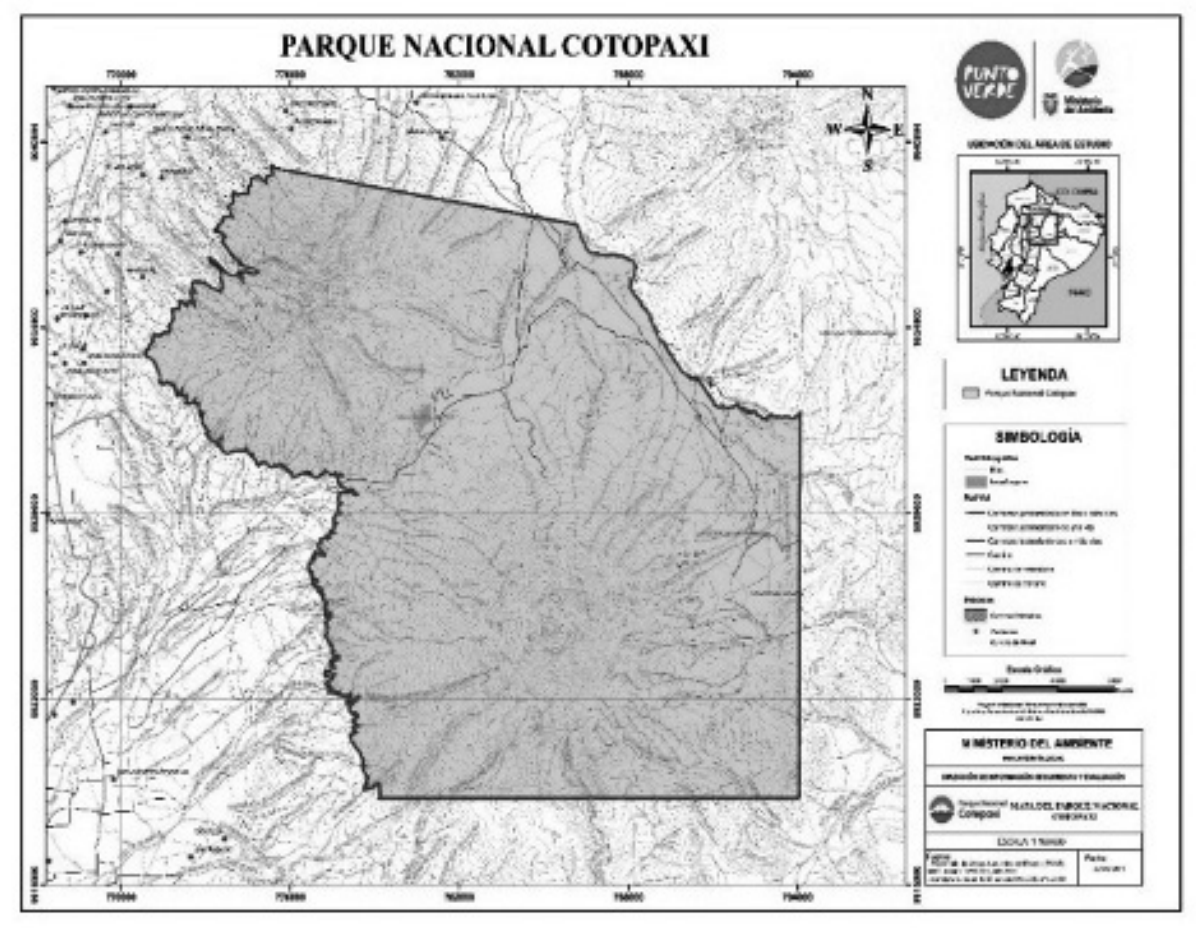

Figura 1. Mapa del Parque Nacional Cotopaxi (MAE, 2015b).

Además, posee otros atractivos como el volcán Rumiñahui y laguna de Limpiopungo. El volcán Rumiñahui es una montaña llena de picos que le dan una apariencia agreste, la cual recuerda al último guerrero indígena que resistió la invasión española y cuyo nombre significa "rostro de piedra". Los picos son paredes de 800 metros de alto que rodean la caldera que colapsó probablemente por el vaciamiento violento de 
la cámara donde se alojaba el magma. La laguna de Limpiopungo se encuentra a 3.800 metros de altitud y cubre 200 hectáreas aproximadamente (MAE, 2015b).

El presente proyecto se basó en una investigación descriptiva; un método científico que involucra observar y describir la situación actual del proyecto con el fin de obtener resultados reales del área de estudio. Se realizó un estudio transversal en el PNC con 374 visitantes a quienes se aplicó una encuesta sobre el perfil y necesidades del turista. Se consideraron los siguientes aspectos en el muestreo:

- Población: todos los visitantes al Parque Nacional Cotopaxi mayores de 18 años que puedan entender la encuesta. 14.142 visitantes mensuales (promedio) según el MAE (2019).

- Población accesible: turistas nacionales y extranjeros que arriban al centro de información del Parque Nacional Cotopaxi.

- Cálculo de la muestra: de acuerdo al tipo de estudio se aplicó una muestra finita, debido a que se conoce el tamaño de la población, con un error del $5 \%$, nivel de confianza del $95 \%$ y una probabilidad de 0,5 , determinando una muestra representativa de 374 unidades primarias de muestreo.

- Asignación aleatoria: para que cada sujeto tenga la misma probabilidad de ser seleccionado, se utilizó un muestreo sistemático (Tapia et al., 2018), en el que se administró una encuesta a cada quinta persona que ingresó al PNC. La muestra se dividió para las 4 semanas de estudio de campo (374/4 = 94 semanales). Criterios de selección: todos los individuos mayores de 18 años que estén en capacidad de responder la encuesta.

Adicionalmente, se realizó una entrevista dirigida a cinco expertos en el área: un representante del Mi- nisterio del Ambiente, un representante del Gobierno Autónomo Descentralizado (GAD), el administrador del Parque Nacional Cotopaxi y 2 guías calificados.

En esta investigación se aplicaron métodos de enfoque cualitativo y cuantitativo. En la recopilación de datos cualitativos se determinó la situación actual del Parque Nacional Cotopaxi basada en una observación de campo, con la aplicación de la "Metodología de los factores geográficos de la actividad turística" (Pazos, 2001).

Para la determinación de los escenarios financieros se utilizaron los deciles para datos no agrupados sobre la intención de gasto, donde cada escenario representa al $10 \%$ de la población. En la tarea de planeación, este punto es una referencia importante, ya que permite determinar un horizonte de mayor proporción de utilidades (Ramírez, 2008).

En cuanto al perfil del turista, se utilizaron encuestas de campo con preguntas previamente establecidas que permitieron recopilar información relevante y precisa, la cual se condensará para la determinación del turista que ingresa al PNC.

Para realizar una propuesta de oferta turística que permita dinamizar la misma, ésta se estructuró con base en el "Manual de operaciones para turismo sostenible en áreas protegidas” (MAE, 2017).

\section{Resultados y discusión}

Para expresar los resultados y discutirlos, es muy importante comenzar por representar la percepción del visitante sobre su experiencia turística en el Parque Nacional Cotopaxi (Tabla 1), la cual, con un rotundo $73 \%$ muestra una percepción positiva del turista hacia el destino.

Tabla 1. Experiencia turística

\begin{tabular}{lccc}
\hline & Frecuencia & Porcentaje & Porcentaje acumulado \\
\hline Excelente & 110 & 29,4 & 29,4 \\
Muy buena & 163 & 43,6 & 73,0 \\
Buena & 93 & 24,9 & 97,9 \\
Regular & 7 & 1,9 & 99,8 \\
Mala & 1 &, 2 & 100,0 \\
Total & 374 & 100,0 & \\
\hline
\end{tabular}


Con un nivel alto de percepción positiva, el segundo paso fue conocer la opinión del acceso a las áreas protegidas, ante lo cual, el 76,2 \% manifiesta que sí debería tener un costo al visitante para el mantenimiento y la protección de estas áreas naturales (Tabla 2).

Tabla 2. Percepción de tarifa de ingreso al PNC

\begin{tabular}{lccc}
\hline & Frecuencia & Porcentaje & Porcentaje acumulado \\
\hline Totalmente de acuerdo & 161 & 43,0 & 43,0 \\
De acuerdo & 124 & 33,2 & 76,2 \\
Indeciso & 29 & 7,8 & 84,0 \\
Desacuerdo & 24 & 6,4 & 90,4 \\
Totalmente en desacuerdo & 36 & 9,6 & 100,0 \\
\multicolumn{1}{c}{ Total } & 374 & 100,0 & \\
\hline
\end{tabular}

Con una percepción positiva y una intención de pago favorable, ahora se identifica el perfil del visitante, cuyas características principales son hombres y mujeres de 35 años de edad, con ingresos económicos mensuales de 1.294 dólares, que visitan 4 veces al año las áreas protegidas y tienen una intención de gasto de 20 dólares por persona (Tabla 3). Este perfil muestra un panorama favorable para poder obtener ingresos por tarifa de usuario, pues es muy importante contrarrestar con la situación del último quinquenio en el que se evidencian aportes técnicos significativos para la sostenibilidad financiera del SNAP, así se concuerda con el Estudio de Necesidades de Financiamiento, el proceso de Valoración Económica de los Bienes y Servicios del Patrimonio de Áreas Naturales del Es- tado y la Estrategia de Sostenibilidad Financiera del SNAP, este último muestra que antes del 2012 (declaratoria de gratuidad de las AP) la autogestión era clave en la sostenibilidad financiera con un aporte estatal de apenas el $35 \%$, pero después de este año pasó a un $94 \%$, dependiendo totalmente de la gestión pública financiera (MAE, 2015a). Además, se coincide con los resultados del análisis de las autoridades de turismo, que destacan una meta financiera estimada en USD 44,1 millones para alcanzar un escenario de consolidación y USD 66,8 millones para lograr un escenario de manejo ideal, de lo cual, en la actualidad el SNAP cuenta con el $48 \%$ de recursos necesarios para alcanzar su consolidación y el $31 \%$ para el escenario ideal de la meta financiera (MAE, 2015a).

Tabla 3. Estadísticos descriptivos del perfil del visitante

\begin{tabular}{|c|c|c|c|c|c|}
\hline & & Edad & Ingreso económico & Frecuencias de visita & Intención de gasto \\
\hline \multirow{2}{*}{$\mathrm{N}$} & Válido & 374 & 364 & 374 & 374 \\
\hline & Perdidos & 0 & 10 & 0 & 0 \\
\hline \multicolumn{2}{|l|}{ Media } & 35,48 & $1.294,61$ & 4,51 & 20,01 \\
\hline \multicolumn{2}{|c|}{ Error estándar de la media } & 0,58 & 70,76 & 0,30 & 1,01 \\
\hline \multicolumn{2}{|l|}{ Mediana } & 34,00 & 925,00 & 3,00 & 15,00 \\
\hline \multicolumn{2}{|l|}{ Moda } & 30 & 1.000 & 2 & 10,00 \\
\hline \multicolumn{2}{|c|}{ Desviación Estándar } & 11,11 & $1.350,05$ & 5,75 & 19,43 \\
\hline \multicolumn{2}{|l|}{ Varianza } & 123,51 & $1.822 .640,13$ & 33,01 & 377,46 \\
\hline \multicolumn{2}{|l|}{ Asimetría } & 0,65 & 2,72 & 3,71 & 1,99 \\
\hline \multicolumn{2}{|c|}{ Error estándar de asimetría } & 0,13 & 0,13 & 0,13 & 0,13 \\
\hline \multicolumn{2}{|l|}{ Mínimo } & 15 & 0 & 1 & 0 \\
\hline \multicolumn{2}{|l|}{ Máximo } & 80 & 10.000 & 50 & 100,0 \\
\hline \multirow{3}{*}{ Percentiles } & 25 & 27,00 & 500,00 & 2,00 & 5,00 \\
\hline & 50 & 34,00 & 925,00 & 3,00 & 15,00 \\
\hline & 75 & 43,00 & 1500,00 & 4,00 & 30,00 \\
\hline
\end{tabular}


Con cifras contundentes sobre la voluntad de pago y la descripción estadística de éstas, se plantearon 10 escenarios financieros, determinados por deciles de una intención de gasto por persona, es decir, cada decil representará al $10 \%$ de la población, agrupando de manera equivalente a la voluntad de pago de 0 a 100 dólares, como se muestra en la Figura 2.

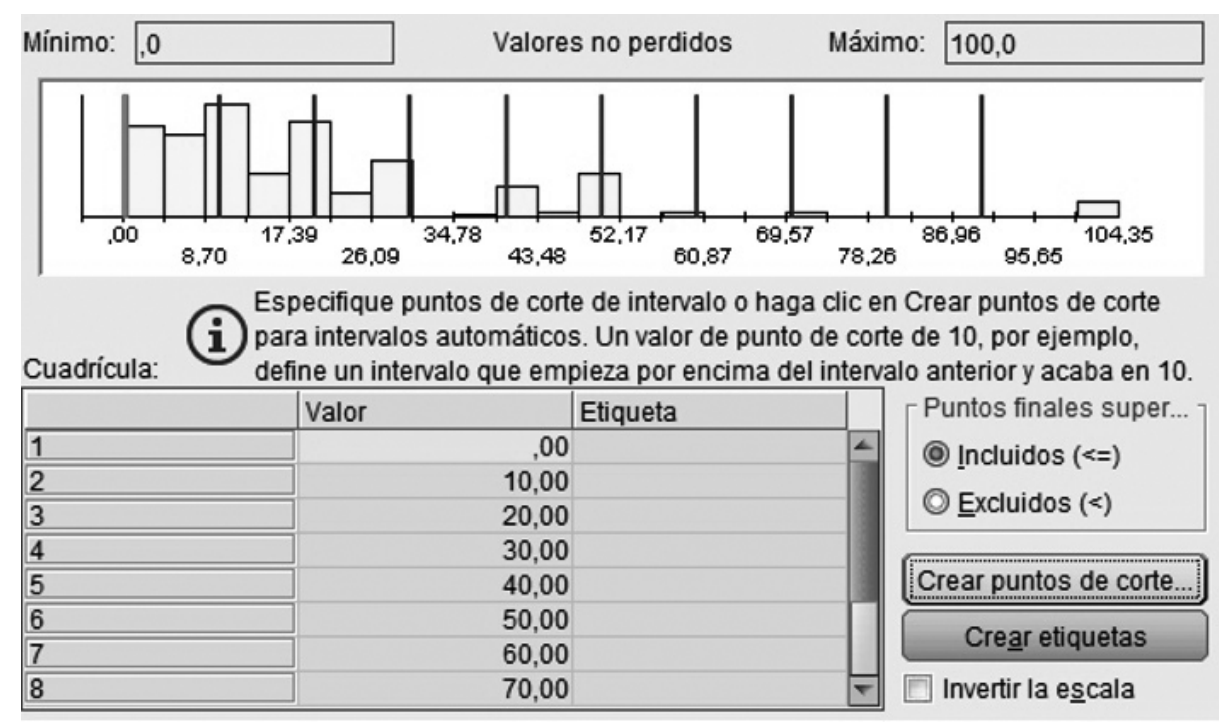

Figura 2. Escenarios financieros por deciles.

Partiendo del punto 0 dólares en color rojo de la gráfica 1 , se determinó que hay tres escenarios financieros óptimos: el primero sería el decil 1 (de 1 a 10 dólares); el segundo sería los deciles 2 y 3 (de 11 a 20; y de 21 a 30 dólares), y el tercero sería el decil 5 (de 41 a 50 dólares); ante lo cual se establece el decil 1 como el escenario 1 solo para tarifa de ingreso al parque, con una media de 8,70 dólares que reajustados con el riesgo implícito sería de 5 dólares para turista nacional y 10 dólares para turista internacional. El segundo escenario son los deciles 2 y 3 , con una media de 21,73 dólares que pueden ajustarse sin problema a los $\mathbf{2 0}$ dólares como el escenario óptimo para una tarifa de usuario nacional, incluyendo la entrada y actividades turísticas; y el tercer escenario corresponde al decil 5 con una media de 43,48 que por la coyuntura del caso puede ajustarse a 50 dólares, como el escenario óptimo para una tarifa de usuario internacional, incluyendo la entrada y actividades turísticas que se describirán más adelante en la turistificación en la tabla 4. Cabe destacar que todos los reajustes se enmarcan dentro de la desviación estándar $\pm 19,4284$.

El planteamiento de estos escenarios es de tal importancia que la misma autoridad ha realizado es- tudios de planificación financiera para las AP, uno de estos se desarrolla en tres escenarios que se proponen alcanzar el equilibrio entre necesidades y fuentes de financiamiento para el SNAP en 10 años. El primer escenario (conservador) pretende cubrir la brecha financiera del escenario de consolidación del SNAP en 4 años y de manejo ideal en 10 años. El segundo escenario (mejorado) consiste en alcanzar el presupuesto necesario para un manejo ideal en un período de 5 años (MAE, 2015a). Cabe resaltar que los primeros dos escenarios suponen que se mantenga el actual modelo de gestión, pero incorporando algunas mejoras. Finalmente, el tercer escenario (matriz productiva) contempla el desarrollo de un nuevo modelo de gestión que se basa en la constitución de una empresa pública (EP) del SNAP (Simancas, 2008).

Por último, es imprescindible la dinamización turística de un destino, es decir que cuente con elementos fundamentales que permitan la diversión plena del visitante. Estos elementos fundamentales se describen como actividades turísticas disponibles: alimentación, tipo de alojamiento mayormente requerido, deportes disponibles para la práctica, facilidades turísticas, transporte dentro del destino, entre otras actividades. En la Tabla 4 se exponen los elementos fundamenta- 
les más requeridos de una extensa gama de opciones consultadas al visitante, mismo que ha sido relacionada con los deciles de los escenarios financieros para de- terminar qué actividades y a qué precio deben fijarse, para de esta manera elaborar un producto específico de dinamización turística del Parque Nacional Cotopaxi.

Tabla 4. Áreas potenciales para la dinamización turística.

\begin{tabular}{|c|c|c|c|c|c|c|}
\hline \multirow{3}{*}{ Actividades } & Deciles & $\begin{array}{c}\text { Espectáculos } \\
\text { culturales }\end{array}$ & Fotografía & $\begin{array}{l}\text { Observación } \\
\text { flora y fauna }\end{array}$ & $\begin{array}{c}\text { Visita } \\
\text { interpretativa }\end{array}$ & $\begin{array}{l}\text { Visitas } \\
\text { guiadas }\end{array}$ \\
\hline & 2 & 42 & 99 & 95 & 68 & 48 \\
\hline & 3 & 32 & 48 & 41 & 45 & 25 \\
\hline \multirow{4}{*}{ Alimentación } & Deciles & $\begin{array}{c}\text { Bebidas } \\
\text { tradicionales }\end{array}$ & $\begin{array}{c}\text { Platos típicos } \\
\text { de la sierra }\end{array}$ & & & \\
\hline & 2 & 34 & 122 & & & \\
\hline & 3 & 24 & 68 & & & \\
\hline & 4 & 14 & 35 & & & \\
\hline \multirow{3}{*}{ Alojamiento } & Deciles & Camping & & & & \\
\hline & 2 & 79 & & & & \\
\hline & 3 & 34 & & & & \\
\hline \multirow[t]{2}{*}{ Deportes } & Deciles & Campismo & $\begin{array}{c}\text { Ciclismo de } \\
\text { montaña }\end{array}$ & Senderismo & Trekking & \\
\hline & 2 & 57 & 85 & 52 & 61 & \\
\hline \multirow[t]{2}{*}{ Facilidades } & Deciles & $\begin{array}{c}\text { Senderos } \\
\text { autoguiados }\end{array}$ & $\begin{array}{l}\text { Senderos } \\
\text { guiados }\end{array}$ & $\begin{array}{l}\text { Servicios } \\
\text { higiénicos }\end{array}$ & & \\
\hline & 2 & 84 & 99 & 106 & & \\
\hline \multirow[t]{2}{*}{ Otros servicios } & Deciles & $\begin{array}{l}\text { Alquiler de } \\
\text { bicicletas }\end{array}$ & $\begin{array}{l}\text { Alquiler de } \\
\text { equipos de } \\
\text { acampada }\end{array}$ & $\begin{array}{l}\text { Punto de } \\
\text { asistencia } \\
\text { médica }\end{array}$ & & \\
\hline & 2 & 83 & 59 & 111 & & \\
\hline \multirow{2}{*}{ Transporte } & Deciles & Bicicletas & Bus turístico & & & \\
\hline & 2 & 81 & 42 & & & \\
\hline
\end{tabular}

Cabe recalcar que se coincide plenamente con todos los estudios previos sobre la necesidad de recursos y cómo financiarlos, pero muy en especial con Rivera \& Vallejos-Romero (2015), quien considera como máximo ejemplo al Parque Nacional Yellowstone, para una dinamización turística con autogestión financiera y cuyo pilar fundamental es la tarifa de ingreso.

\section{Conclusiones}

La declaratoria de gratuidad de las áreas protegidas, determinó una dependencia financiera del Estado central, coadyuvando al escaso desarrollo turístico del Parque Nacional Cotopaxi.

Se determinaron tres escenarios financieros óptimos en función de los deciles de la intención de gasto, que incluye la tarifa de ingreso para el visitante nacional de 5 dólares e internacional de 10 dólares, y la tarifa con elementos fundamentales de turistificación para el turista nacional en 20 dólares y para el internacional en 50 dólares.

Los elementos fundamentales para la dinamización turística están determinados en los escenarios financieros 2 y 3 para el turista nacional y 5 para el turista internacional, de aquí que, una buena oferta turística determinará la autosustentabilidad del Parque Nacional Cotopaxi.

\section{Agradecimientos}

Un profundo agradecimiento a la Dirección General de Investigación de la Universidad Central del Ecuador, por el apoyo brindado en todas las 
fases de ejecución del proyecto de investigación. También es imperante reconocer y agradecer a las autoridades del Ministerio del Ambiente y del Parque Nacional Cotopaxi por la apertura y apoyo a los investigadores.

\section{Referencias}

Acuerdo Ministerial No. 006. Gratuidad en Áreas protegidas.16 de enero del 2012 (Ecuador).

Cifuentes A., M., Izurieta V., A., \& Faria, H. H. (2000). Medición de la efectividad del manejo de las áreas protegidas. Serie técnica /WWF $\mathrm{N}^{\circ} 2$. WWF-Central America, IUCN, Deutsche Gesellschaft für Technische Zusammenarbeit (GTZ).

Constitución de la República del Ecuador [Const] Art. 37, 71, 405. 20 de octubre de 2008.

Dudley, N. (Editor) (2008). Directrices para la aplicación de las categorías de gestión de áreas protegidas. Gland. Suiza: UICN

Lausche, B. J. (2011). Guidelines for protected areas legislation. IUCN Environmental Policy and Law Paper, $N^{\circ}$ 081. Gland. Suiza: UICN

Ley de Turismo. Ley 97. Art. 20. 29 de diciembre de 2014.

Maldonado, E. R. (2000). Determinación de la capacidad de carga turística, como una opción para el manejo sustentable del Parque Nacional La Tigra, Tegucigalpa, Honduras. CATIE (Centro Agronómico Tropical de Investigación y Enseñanza).

Ministerio del Ambiente [MAE]. (2015a). Estrategia de Sostenibilidad Financiera del Sistema Nacional de Áreas Protegidas (SNAP) del Ecuador. MAE, PNUD.

Ministerio del Ambiente [MAE]. (2015b). Sistema nacional de áreas protegidas del Ecuador. Obtenido de http://areasprotegidas.ambiente.gob. ec/info-snap

Ministerio del Ambiente [MAE]. (2017). Manual de operaciones para turismo sostenible. Áreas

\section{Financiamiento}

Este proyecto de investigación fue financiado totalmente por el Fondo Semilla 5 de la Dirección de Investigación de la Universidad Central del Ecuador.

protegidas de Cuyabeno, Limoncocha, Yasuní. MAE, Deutsche Gesellschaft für Technische Zusammenarbeit (GTZ).

Ministerio del Ambiente [MAE]. (2018). Áreas Protegidas. Obtenido de http://www.ambiente.gob. ec/areas-protegidas-3/

Ministerio del Ambiente [MAE]. (2019). Reportes históricos de visitas. Obtenido de http:// areasprotegidas.ambiente.gob.ec/es/reporte-de-visitas\#

Nel-lo Andreu, M. (2008). La intensidad de la gestión. La clave para un desarrollo turístico sostenible en áreas protegidas. Diagnóstico de las áreas protegidas de América Central. Boletín de la Asociación de Geógrafos Españoles, 47, 333-354.

Pauchard, A., \& Villarroel, P. (2002). Protected areas in Chile: History, current status, and challenges. Natural Areas Journal 22(4), 318-330.

Pazos, C. (2001). Documento pedagógico Patrimonio Turístico Nacional I Geografía. Universidad de Especialidades Turísticas.

Ramírez Padilla, D. N. (2008), Contabilidad Administrativa. McGraw Hill.

Reglamento especial de turismo en áreas naturales protegidas. Decreto Ejecutivo 827. 19 de enero de 2016 (Ecuador).

Rivera, C., \& Vallejos-Romero, A. (2015). La privatización de la conservación en Chile: repensando la gobernanza ambiental. Bosque (Valdivia), 36(1), 15-25. https://dx.doi.org/10.4067/S071792002015000100003 
Román Márquez, A. (2011). Planes de dinamización y excelencia turística versus municipios turísticos; problemática, similitudes y diferencias escenciales. Papers de turisme, 49$50,25-44$.

Secretaría Nacional de Planificación y Desarrollo [Senplades]. (2017). Plan Nacional de Desarrollo 2017-2021-Toda una Vida.Senplades. http://www.planificacion.gob.ec/wp-content/ uploads/downloads/2017/10/PNBV-26-OCTFINAL_0K.compressed1.pdf

Sepúlveda, C., \& Villarroel, P. (2006) Servicios ecosistémicos y financiamiento de la conservación privada en Chile. Ambiente y Desarrollo, 22, 12-20.
Simancas Cruz, M. R. (2008). El sistema de cobro de una tasa pública por la prestación de servicios turísticos en las áreas protegidas de Canarias. Cuadernos De Turismo, 21, 201-237.

Tapia Novillo, F. C., Díaz Muñoz, D. J., Sandoval Cantuña, L. S. Tapia Calderón, E. P., \& Pazmiño Valle, P. M. (2018), Competitividad aérea intercontinental: Análisis de los precios de los tickets aéreos y la distancia entre destinos. Revista Espacios, 39(51), 12.

Vergara Schmalbach, J. C., Fontalvo Herrera, T. J., \& Maza Ávila, F. M. (2010). La planeación por escenarios: Revisión de conceptos y propuestas metodológicas. Prospect, 8(2), 21-29. 